\title{
Suppression of Non-Small Cell Lung Cancer Growth and Metastasis by a Novel Small Molecular Activator of RECK
}

\author{
Jia Shen ${ }^{\text {b, d }}$ Banghua Wang ${ }^{\mathrm{a}}$ Tao Zhang ${ }^{\mathrm{a}} \quad \mathrm{Ni}_{\text {Zhu }}^{\mathrm{a}}$ Zexia Wang ${ }^{\mathrm{a}}$ \\ Jing Jinc Yi Hec Meichun $\mathrm{Hu}^{\mathrm{a}}$
}

${ }^{a}$ Research Center of Basic Medical Sciences, School of Basic Medical Sciences, Hubei University of Science and Technology, Xianning, 'binstitute of Biochemistry and Cell Biology, Shanghai Institutes for Biological Sciences, Chinese Academy of Sciences, Shanghai, 'Department of Urology, The First Affiliated Hospital of Jiaxing College, Jiaxing, China, dPresent address: Tumor Initiation and Maintenance Program, Sanford Burnham Prebys Medical Discovery Institute, La Jolla, California, USA

\section{Key Words}

Harmine $・$ RECK $・$ NSCLC $・$ Apoptosis $・$ Metastasis

\begin{abstract}
Background/Aims: Reversion-inducing cysteine-rich protein with kazal motifs (RECK) is a novel tumor suppressor gene that is critical for regulating tumor cell invasion and metastasis. The expression of RECK is dramatically down-regulated in human cancers. Harmine, a tricyclic compound from Peganum harmala, has been shown to have potential anti-cancer activity. Methods: Cell proliferation assay (CCK-8 cell viability assay), cell cycle analysis (detection by flow cytometry), apoptosis staining assay (TUNEL staining), cell migration assay and invasion assay (transwell assay) were carried out to investigate the Harmine's efficacy on non-small cell lung cancer (NSCLC) cells in vitro. A549-luciferase cell orthotropic transplantation xenograft mouse model was used to determine the effect of Harmine treatment on NSCLC in vivo. Western blotting analysis of cell growth and metastasis related signal pathways was conducted to investigate the molecular mechanism of Harmine's inhibitory effect on NSCLC. Results: Harmine treatment effectively inhibited cell proliferation and induced the G1/S cell cycle arrest of NSCLC cells. Further study proved that Harmine treatment led to apoptosis induction. Furthermore, treatment with NSCLC cells with Hamine resulted in decreased cell migration and cell invasion in vitro. More importantly, Harmine treatment significantly suppressed the NSCLC tumor growth and metastasis in mouse xenograft model in vivo. Mechanistically, in Harmine-treated NSCLC cells, RECK expression and its downstream signaling cascade were dramatically activated. As a consequence, the expression level of MMP-9 and E-cadherin were significantly decreased. Conclusion: These findings identify Harmine as a promising activator of RECK signaling for metastatic NSCLC treatment.




\section{Cellular Physiology Cell Physiol Biochem 2018;45:1807-1817

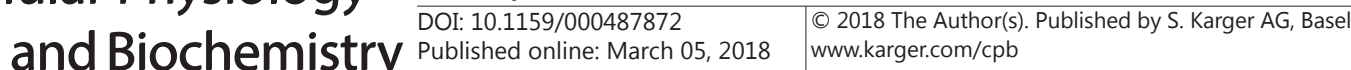 \\ Shen et al.: Harmine Suppresses NSCLC by Activating RECK Signaling}

\section{Introduction}

Reversion-inducing cysteine-rich protein with Kazal motifs (RECK) gene expresses in all human normal tissues but is down-regulated in a variety of tumor tissues including liver cancer [1], gastric cancer [2] and colorectal cancer [3]. RECK protein blocks the tumor metastasis and invasion via negative regulation of matrix metalloproteinases (MMPs) [4]. MMPs are important enzymes in the tumor microenvironment associated with progression of many kinds of cancers [5-7]. Indeed, there is a significant imbalance between MMPs and RECK, which serves as an important mechanism contributing to tumor progression with high grade lesions [8]. Many reports identified the oncogenic role of the protein signal transducer and the activator of transcription 3 (STAT3) in cancers, and STAT3 hyperactivation are known to be fundamentally important for metastasis [9-12]. Previous study reported RECK can control cancer metastasis by modulating STAT3-dependent neoangiogenic switch and STAT3 activation [13]. Meanwhile, MMPs are the direct transcriptional target genes of STAT3, which up-regulates MMP2 and MMP9 expression that promotes cancer metastasis. Thus there is a feedback loop between RECK, STAT3 and MMPs, suggesting the up-expression of the tumor suppressor RECK may influence the signal transduction of STAT3 and MMPs. Notably, several previous studies reported that up-regulation of RECK resulted in decreased ovarian cancer amplification [14]. In addition, histone deacetylase inhibitor inhibited ovarian cancer cell migration partially through up-regulation of RECK [15].

Lung cancer remains the leading cause of cancer-related mortality worldwide although the large number of clinical trials aimed at improving patients' survival [16-18]. More than 1.5 million new cases of lung cancer are diagnosed every year, approximately $80 \%$ of which are NSCLC [19-21]. With regards to lung cancer mortality, $80 \%-90 \%$ of these cases are associated with metastasis. Importantly, metastasis is the critical step in malignant tumor progression and the main cause of treatment failure. Therefore, interfering with the tumor cell metastatic cascade might be an attractive treatment option to counteract metastatic tumor dissemination. Currently, chemotherapy plays a significant role and is commonly used in comprehensive NSCLC therapy, however the toxic side effects must be attenuated in order to reap the maximum benefits. Therefore, more attentions have been focused on natural compounds with minimal or no toxicity and having the strong efficacy against NSCLC cells.

Harmine, a major constituent in a hallucinogenic botanical mixture ayahuasca and medical plant Peganum harmala, is a tricyclic compound belonging to the $\beta$-carboline alkaloids [22]. In latest studies, Harmine has been proved to inhibit the growth of ovarian cancer in vitro [23]. However, the function and mechanism underlying the antitumor activity of Harmine against NSCLC remain unknown. The present study was designed to evaluate whether Harmine alters metastatic tumor cell progression in vitro and in vivo. Our results demonstrated that Harmine induced G1/S phase cell cycle arrest and apoptosis in NSCLC cells. Further study suggested that Harmine treatment led to inhibition of cell metastasis and invasion properties of NSCLC cells. Moreover, Harmine suppressed xenograft of A549 tumor growth and metastasis in vivo, suggesting that Harmine is a promising candidate for metastatic NSCLC treatment.

Previous study reported expression of RECK in NSCLC was significantly lower than in the normal lung tissue [24]. Furthermore, assessing the RECK protein, Takenaka et al. observed a higher 5-year survival rate for cancer patients with tumors with strong RECK expression [25, 26]. Hence re-activation of RECK expression and its related signaling pathways by natural compounds is a rational strategy for the treatment of NSCLC.

\section{Materials and Methods}

Cell lines, animals and reagents

Human NSCLC cell lines H460, A549, PC9 and normal human fetal lung fibroblast MRC-5 cells were purchased from ATCC. All cells were incubated at $37{ }^{\circ} \mathrm{C}$ with $5 \%$ humidified $\mathrm{CO}_{2}$. A549 cells transfected 


\section{Cellular Physiology Cell Physiol Biochem 2018:45:1807-1817

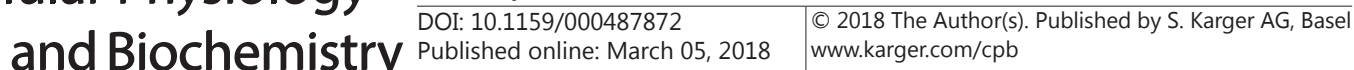 \\ Shen et al.: Harmine Suppresses NSCLC by Activating RECK Signaling}

with pGL4 vector (Promega) expressing luciferase were selected in G418 to produce the stable A549-luc cell line. Mice were obtained from National Rodent Laboratory Animal Resources, Shanghai Branch of China. Harmine with a purity of up to $98 \%$ was purchased from Shanghai Yuanye Biotechnology Co., Ltd. Harmine was dissolved in DMSO (Sigma) at a stock solution of $100 \mathrm{mM}$ and stored at $-20^{\circ} \mathrm{C}$. Antibodies were purchased from Cell Signaling Technology.

\section{Cell cycle distribution analysis}

Cell cycle analysis was performed according to previous report [27]. Cells were treated with four different doses $(0,5,10,20 \mu \mathrm{M})$ of Harmine for $24 \mathrm{~h}$. The cells were fixed in $70 \%$ ethanol at $-20{ }^{\circ} \mathrm{C}$ for $2 \mathrm{~h}$, washed in PBS and stained with propidium iodide (PI, $50 \mu \mathrm{g} / \mathrm{mL}$ ) containing ribonuclease A (50 $\mathrm{ng} / \mu \mathrm{L})$ at $37^{\circ} \mathrm{C}$ for $1 \mathrm{~h}$. Then cells were analyzed by flow cytometry (FACSCalibur; BD Biosciences).

\section{Cell viability assay}

Human NSCLC and MRC-5 cells seeded in 96-well plates were subjected to indicated treatment. Cell viability detection was carried out by using a CCK-8 detection kit (Dojindo Molecular Technologies, Inc., Gaithersburg, MD) according to the manufacturer's instructions as described before [19].

\section{Apoptosis TUNEL assay}

TUNEL (Terminal deoxynucleotidyl transferase dUTP nick end labeling, TUNEL) assay is a method for detecting apoptotic DNA fragmentation, and widely used to identify and quantify apoptotic cells, or to detect excessive DNA breakage in individual cells. In Situ Cell Death Detection Kit (Fluorescein) that was produced by Roche company was utilized for apoptosis TUNEL assay (Cat No. 11684795910). Simply, cells after treatment of Harmine for $36 \mathrm{~h}$ were fixed overnight in 4\% (v/v) paraformaldehyde in PBS at room temperature, then washed 3 times with PBS, treated with Proteinase K (20 ng/mL, Invitrogen) in $10 \mathrm{mM}$ Tris-HCl (pH 7.5) at $37^{\circ} \mathrm{C}$ for $30 \mathrm{~min}$, and then washed 3 times with PBS. TUNEL reaction was performed in a microcentrifuge tube $(1.5 \mathrm{~mL})$ using the In Situ Cell Death Detection Kit according to the manufacturer's instructions.

\section{Western blotting}

Cells were exposed to drug treatment for indicated time and lysed in RIPA buffer as detailed in previous report [28]. Lysate was mixed with sample loading buffer and heated at $100^{\circ} \mathrm{C}$ for $10 \mathrm{~min}$. Whole samples were run on $8 \%$ to $12 \%$ SDS-PAGE gels. Then extracted protein were transferred to PVDF membranes. Then the membranes were blocked with $5 \%$ skim milk before incubated with specific primary antibodies overnight at $4^{\circ} \mathrm{C}$.

\section{Migration assay}

Wound-healing migration assay and chamber migration assay were conducted to examine the effect of Harmine treatment on NSCLC cell migration as described before [29]. Briefly, A549 cells were seeded into 6-well plates with $100 \%$ confluence, then the "wounds" were created by a sterile pipette tip. Fresh medium containing various concentrations of Harmine was added. After $12 \mathrm{~h}$ incubation, cells were fixed with 3.7\% paraformaldehyde. After that, the migrated cells were photographed and quantified. In additon, cell migration assay by Transwell/Boyden chambers ( $8 \mu \mathrm{m}$; BD Biosciences) was also performed. Serumstarved A549 cells in $100 \mu \mathrm{L}$ medium with $0.5 \%$ FBS were pretreated with Harmin for 30 min before seeded on the upper chamber of Transwell. Then the cells migrated to the lower chamber with $600 \mu \mathrm{L}$ medium. After 5 to $7 \mathrm{~h}$ incubation, non-migrated cells were removed with cotton swabs, and migrated cells were fixed with ice-cold 3.7\% paraformaldehyde and stained with $0.1 \%$ crystal violet. Images were taken with an inverted microscope and migrated cells were quantified.

\section{Invasion assay}

Invasion assay was performed using Transwell/Boyden chambers coated with Matrigel as described previously [29]. In brief, A549 cells were pretreated with indicated concentrations of Harmine in 6-well plates for $12 \mathrm{~h}$. A total of $5 \times 10^{4}$ cells in $100 \mu \mathrm{L}$ of serum free medium were added in the upper chamber, and $600 \mu \mathrm{L}$ of complete medium were added in the bottom chamber. Various concentrations of Harmine were added to both chambers. The A549 cells were allowed to invade for $12 \mathrm{~h}$ at $37^{\circ} \mathrm{C}$. Then the invaded cells were stained with $0.1 \%$ crystal violet and counted manually. 


\section{Cellular Physiology Cell Physiol Biochem 2018:45:1807-1817 \begin{tabular}{l|l} 
and Biochemistry POI: 10.1159/000487872 & $\begin{array}{l}\text { (c) } 2018 \text { The Author(s). Published by S. Karger AG, Basel } \\
\text { www.karger.com/cpb }\end{array}$
\end{tabular} \\ Shen et al.: Harmine Suppresses NSCLC by Activating RECK Signaling}

A549-luciferase cell orthotropic transplantation xenograft model

A549-Luciferase cells $\left(5 \times 10^{5}\right)$ in $0.02 \mathrm{~mL}$ liquids that were mixed with PBS and Matrigel were orthotopically transplantated into the lung of nude mice after anaesthetized by pentobarbital sodium. Seven days later, based on photon flux indexes detected by Xenogen IVIS 2000 Luminal Imagier, all mice bearing tumor were devided into three groups ( $n=10$ per group) randomly and from that day on, the luminal photos were taken every 7 days and photon flux indexes which could represent the orthotopic tumor sizes were recorded every 7 days. Harmine $(10 \mathrm{mg} / \mathrm{kg} /$ day per mouse and $20 \mathrm{mg} / \mathrm{kg} /$ day per mouse) was injected intraperitoneally every day. The control group was treated with dimethyl sulfoxide. Twenty eight days later, the mice were sacrificed. The growth rate curve of the tumor xenograft was evaluated by determining the photon flux indexes. The mouse body weight was measured every 4 days. Lung tumor xenografts were fixed and prepared for immunohistochemistry.

\section{Immunohistochemistry analysis}

Immunohistochemistry was performed as previously reported [29]. Primary tumors and mets were excised, fixed and embedded in paraffin. To examine the effect of Harmine treatment on cancer cells proliferation and apoptosis in vivo, sections $(4 \mu \mathrm{m})$ were stained with Ki-67, RECK and MMP9. Images were obtained with Leica microscope (Leica, DM4000b).

\section{Statistical analysis}

Results were statistically analyzed using the Student's $t$ test with Microsoft Excel except for the mets statistical result, which was analyzed using GraphPad Prism version 4.02. All experiments were independently repeated at least three times. A value of $P<0.05$ was considered statistically significant. For all graphs: ${ }^{*} \mathrm{P}<0.05 ;{ }^{* *} \mathrm{P}<0.01 ;{ }^{* * *} \mathrm{P}<0.001$.

\section{Results}

\section{Cytotoxicity of Harmine on NSCLC cells}

The chemical structure of Harmine was shown in Fig. 1. To evaluate the cytotoxicity of Harmine on human NSCLC cells (A549, H460 and PC9 cells) and further examine whether Harmine had ideal selective effects between NSCLC cells and normal human fetal lung fibroblasts (MRC-5 cells), cells were treated with various concentrations of Harmine for 48 $\mathrm{h}$, then cell viability was assessed with CCK-8 assay. As shown in Table 1, the half-maximum inhibiting concentrations ( $\mathrm{IC}_{50}$ value) of Harmine on NSCLC cells ranged from $10.11 \mu \mathrm{M}$ to $32.74 \mu \mathrm{M}$, but the toxicity on MRC-5 cells was negligible, indicating Harmine had appreciable selectivity between normal human lung cells and cancer cells. Furthermore, Fig. 2A and 2B illustrated that Harmine selectively inhibited NSCLC A549 cell proliferation in a time- and dose-dependent manner.

\section{Harmine treatment induced G1/S phase arrest and} apoptosis in NSCLC cells

To further investigate whether Harmine could influence cell cycle of NSCLC cells, the A549 cells were exposed to different concentration of Harmine for $24 \mathrm{~h}$ and cell cycle distribution analysis was carried out by flow cytometry. As shown in Fig. 2C, compared to the control group, a remarkable increase of G1 phase population was observed in Harmine-treated cells in a dose-dependent manner. Then we set out to investigate whether Harmineinduced cell cycle arrest could cause apoptosis of A549 cells. The TUNEL staining analysis in A549 cells after Harmine treatment revealed that Harmine treatment led to cell apoptosis induction in A549 cells (Fig. 2D). Taken together, these results suggest Harmine treatment caused G1/S phase arrest and apoptosis of A549 cells.

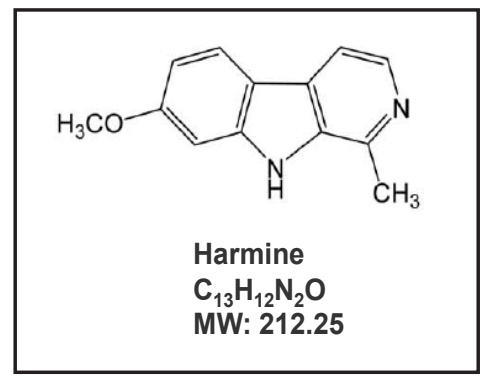

Fig. 1. (A) Chemical structure and molecular weight of Harmine. Table 1. The half maximal inhibitory concentration $\left(\mathrm{IC}_{50}\right)$ of Harmine in normal human fetal lung fibroblast MRC-5 cells and different NSCLC cells. 


\section{Cellular Physiology Cell Physiol Biochem 2018;45:1807-1817 \begin{tabular}{l|l} 
and Biochemistry Published online: March 05, 2018 & $\begin{array}{l}\text { (c) } 2018 \text { The Author(s). Published by S. Karger AG, Basel } \\
\text { www.karger.com/cpb }\end{array}$
\end{tabular}

Harmine inhibits migration and invasion of NSCLC cells In lung cancers, metastasis-related recurrence is still common and responsible for the cancer-associated mortality [30]. To determine the effect of Harmine on NSCLC metastasis, we performed cell migration and invasion assays using A549 cells that have highly malignant mobility. Specifically, compared with the control group, the wound size exhibited a remarkably reduced trend after $2.5 \mu \mathrm{M}$ Harmine treatment, implying that the treatment of Harmine could weaken the cell horizontal migration activity (Fig. 3A). Likewise, Harmine also impaired the vertical migration ability of A549 cell through the Transwell results (Fig. 3B). Additionally, Harmine exhibited a modest inhibitory effect on NSCLC cells invasion, which was also an important indicator of tumor biological activities. The result showed that $5 \mu \mathrm{M}$ Harmine almost absolutely blocked cell invasion (Fig. 3C). In sum, Harmine significantly inhibited A549 cell wound healing, migration and invasion in a dosedependent manner.

Harmine inhibits A549 tumor growth and metastasis in vivo

To further evaluate the tumorsuppressing effect of Harmine in vivo, a model for tumorigenicity of NSCLC cells in nude mice was established. First, a luciferase stably expressing A549 cell line (A549-luc) was established by transfecting the A549 cells with luciferase-expressing plasmids. Upon orthotopic injection into mice lungs from day 0-28, A549-luc cells exhibit bioluminescence traced by photon flux indexes (Fig. 4A). Mice were randomly divided into 3 groups ( $n=10$ per group) and treated with Harmine at $10 \mathrm{mg} /$ $\mathrm{kg} /$ day or $20 \mathrm{mg} / \mathrm{kg} /$ day or vehicle control. Tumors in each mouse were monitored and imaged by IVIS instrument every 7 days to examine local tumor growth and tumor cells dissemination. As shown in Fig. $4 \mathrm{~A}$ and $4 \mathrm{~B}$, tumor cells with high levels of bioluminescence were clearly detected in the lung region as well as other parts of the body of the control mice, which increased remarkably as time went by. In
Table 1. The half maximal inhibitory concentration (IC) of Harmine in different lung cells

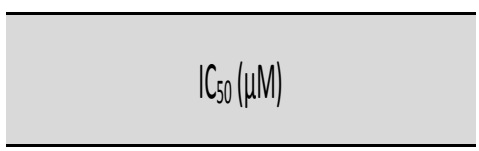

MRC.5 A549 H460 PC9 $>50 \quad 10.11 \quad 19.45 \quad 32.74$

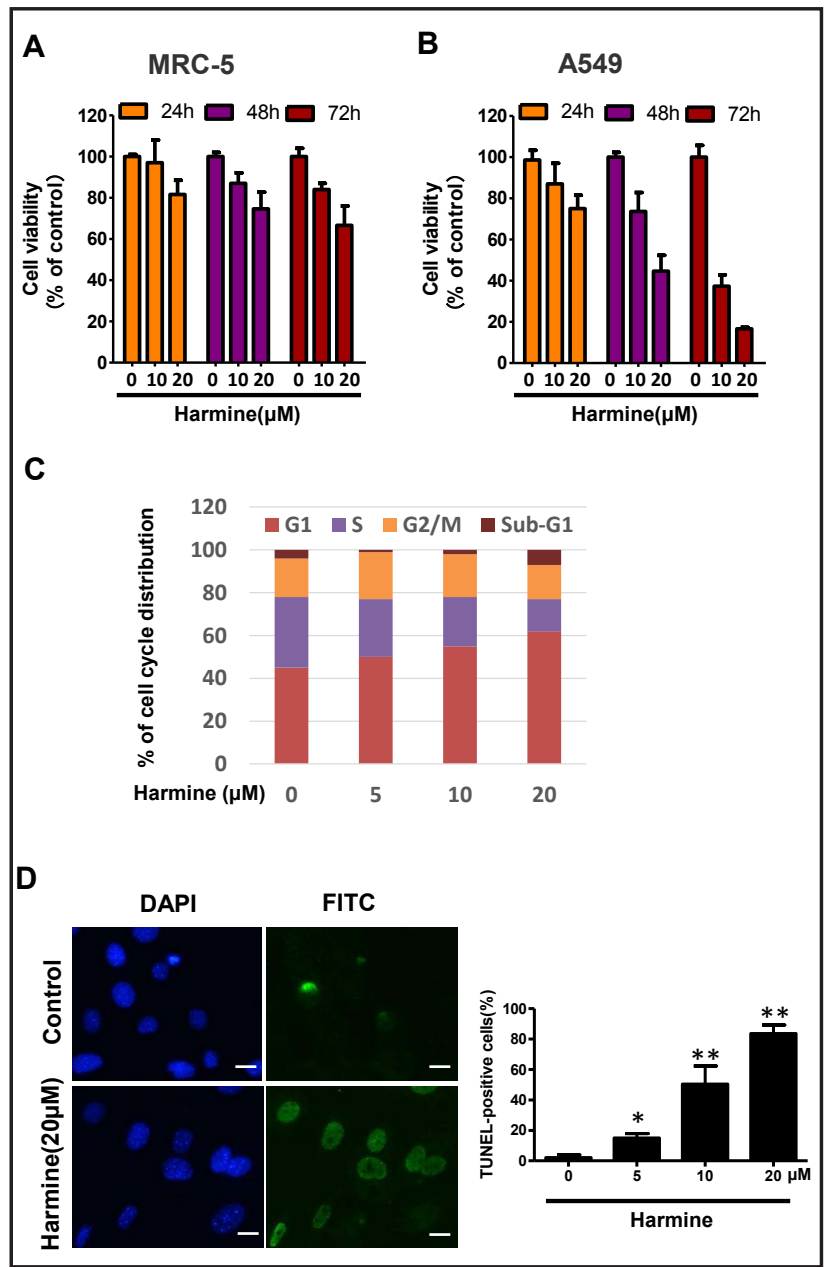

Fig. 2. Cell viability assay was performed to investigate Harmine's efficacy on MRC-5 cells (A) and NSCLC A549 cells (B) at indicated concentrations and hours. Data were presented as mean \pm SEM., $n=3$. (C) Harmine induced cell cycle arrest on NSCLC A549 cells. Cells were analyzed by flow cytometry after treatment with Harmine for $24 \mathrm{~h}$. (D) Representative images (left panel) and quantification chart (right panel) of apoptotic analysis by TUNEL staining in A549 cells after Harmine treatment. Scale bars represent $10 \mu \mathrm{m}$. 


\section{Cellular Physiology and Biochemistry}

Cell Physiol Biochem 2018;45:1807-1817

\begin{tabular}{l|l}
\hline DOI: $10.1159 / 000487872$ & C 2018 The Author(s). Published by S. Karger AG, Basel
\end{tabular}

Shen et al.: Harmine Suppresses NSCLC by Activating RECK Signaling
Fig. 3. Effects of Harmine on migration and invasion of A549 cells in vitro. (A) NSCLC A549 cells were allowed to migrate cross a "wound" when treated with Harmine (from $0 \mu \mathrm{M}$ to $5 \mu \mathrm{M}$ ) for $12 \mathrm{~h}$. The number of migrated cells were calculated. (B) A549 cells were seeded on the upper chamber of Transwell. After 5 to $7 \mathrm{~h}$ treatment with Harmine at indicated concentrations (from 0 $\mu \mathrm{M}$ to $5 \mu \mathrm{M}$ ), migrated cells were fixed, stained and counted. (C) A549 cells were treated with Harmine (from $0 \mu \mathrm{M}$ to $5 \mu \mathrm{M}$ ) for $12 \mathrm{~h}$ and seeded in the upper chamber of Transwell coated with Matrigel to invade for another $12 \mathrm{~h}$. Invaded cells were fixed, stained and counted. All data are represented as mean \pm S.D. from triplicate wells. ${ }^{*} \mathrm{p}<0.05,{ }^{* *} \mathrm{p}<0.01,{ }^{* * *} \mathrm{p}<0.001$, as compared to control.

Fig. 4. In vivo anticancer effects of Harmine on NSCLC mouse xenograft models. (A) Representative in vivo bioluminescence images of mice bearing A549-luc tumors. The tumor model was established according to the steps described in Materials and Methods. (B) Quantification of whole-body bioluminescence in control and Harmine treatment groups. (C) Representative photos of bioluminescence detection for lung regions excised from control and Harmine-treated tumor-bearing nude mice. (D) Quantification of mets in each mouse in control and Harmine treatment groups. The means and 95\% confidence intervals (error bars) were presented ( ${ }^{* * *} \mathrm{P}<0.001$, ${ }^{* *} \mathrm{P}<0.01,{ }^{*} \mathrm{P}<0.05$ ). (E) Mice body weight was measured during Harmine treatment $(n=10$; one-way ANOVA statistic test was used).
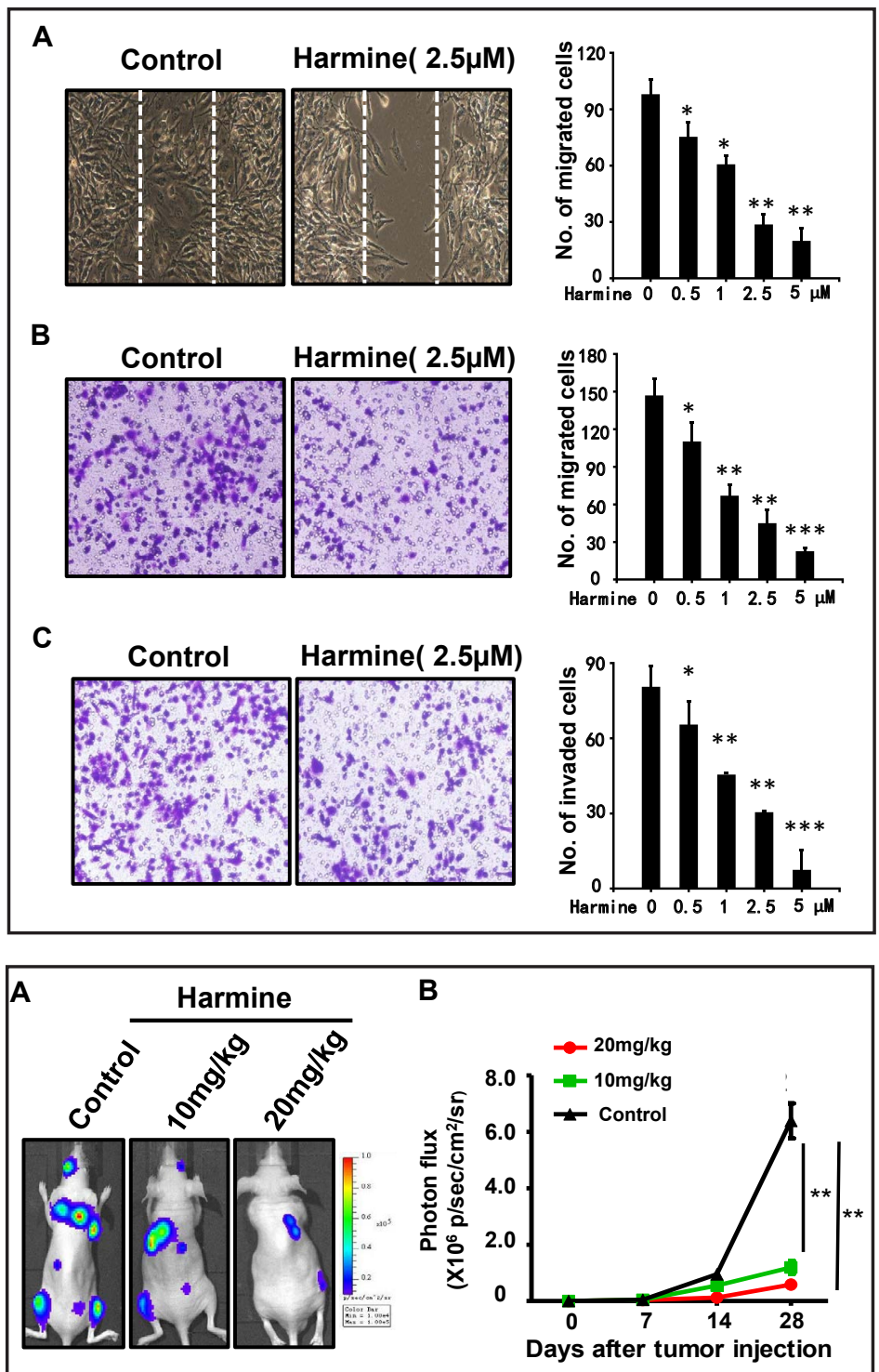

C

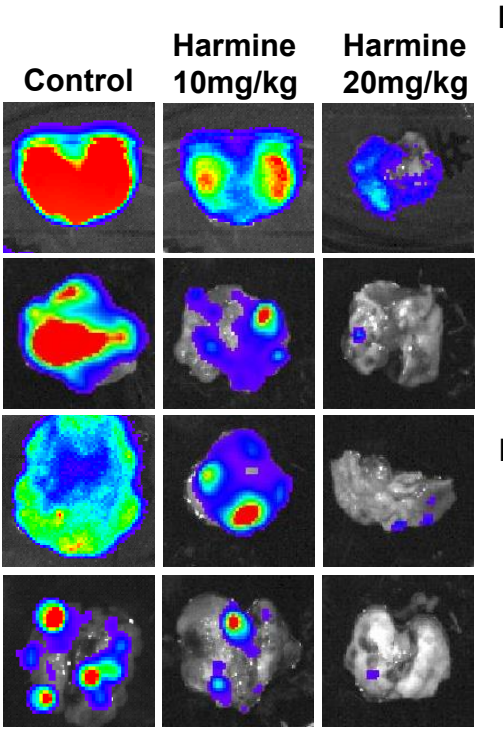

D

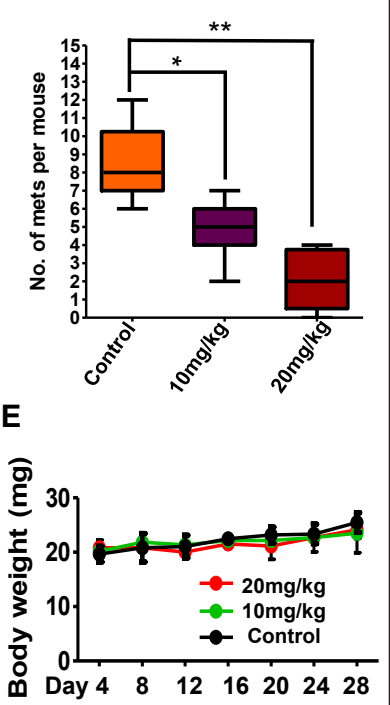


contrast, treatment with Harmine statistically reduced the photo flux indexes in the mice. At day 28 the nude mice were sacrificed. As shown in Fig. 4B, the average normalized photon flux of the $10 \mathrm{mg} / \mathrm{kg} /$ day Harmine-treated group and $20 \mathrm{mg} / \mathrm{kg} /$ day Harmine-treated group was $0.98 \times 10^{6} \pm 0.06 \mathrm{p} / \mathrm{sec} / \mathrm{cm}^{2} / \mathrm{sr}$ and $0.37 \times 10^{6} \pm 0.04 \mathrm{p} / \mathrm{sec} / \mathrm{cm}^{2} / \mathrm{sr}$, respectively, while that of vehicle control group was $6.2 \times 10^{6} \pm 0.22 \mathrm{p} / \mathrm{sec} / \mathrm{cm}^{2} / \mathrm{sr}$. The representative photos of bioluminescence detection for lung regions excised from control and Harminetreated tumor-bearing nude mice were shown in Fig. 4C. Furthermore, we dissected the mice following sacrifice. Compared to the control group, Harmine significantly decreased metastatic nodes numbers in target organs (Fig. 4D). Notably, after 28 days' treatment, the result showed that Harmine treatment at the given concentration had little effect on the body weight of the mice (Fig. 4E). In summary, these in vivo results are consistent with our in vitro results and indicate that administration of Harmine therapeutically blocked A549 tumor growth and metastasis.

\section{Harmine treatment activates RECK signaling pathway in A549 cells}

Next we sought to explore the molecular mechanism by which Harmine inhibits NSCLC cells. Since RECK plays an important role in cancer metastasis and invasion, we detected the mRNA expression levels of RECK in five NSCLC cell lines (H596, A549, H460, H1299 and PC9) and in normal lung cell line (MRC-5) by RT-PCR. The results showed that RECK was significantly down-regulated in NSCLC cell lines, compared with that in normal cells (Fig. $5 A)$. Interestingly, both the mRNA expression level and protein level of RECK were induced upon Harmine treatment in a dose-dependent manner in A549 cells (Fig. 5B and 5C). We further examined the protein expression of STAT3, AKT, ERK, MMP9 and EMT marker E-cadherin upon Harmine treatment. As shown in Fig. 5D, in Harmine-treated A549 cells, western blotting revealed a significant decrease of p-STAT3, AKT, ERK and MMP9 expression, compared with control group. Inversely, the expression level of E-cadherin was higher in Harmine-treated group than control group, suggesting Harmine treatment led to activation of cellular metastatic related signaling pathways. Accordingly, using the cell proliferation assay, decreased viability of A549 cells induced by Harmine treatment was reversed after

Fig. 5. Harmine activates the RECK signaling pathway in NSCLC cells. (A) RTPCR detection of RECK gene expressions in MRC-5 cells and various NSCLC cell lines. RT-PCR detection (B) and Western blotting detection (C) of RECK gene expression upon Harmine treatment in A549 cells. (D) Western blotting analysis of several signaling pathways under Harmine treatment. (E) A549 cells were transiently transfected with RECK siRNA or the control siRNA for $48 \mathrm{~h}$ and the knockdown effect was detected by Western blotting. Cells were treated with Harmine $(0,10$ and $20 \mu \mathrm{M})$ for another $48 \mathrm{~h}$ and the cell viability was assessed.

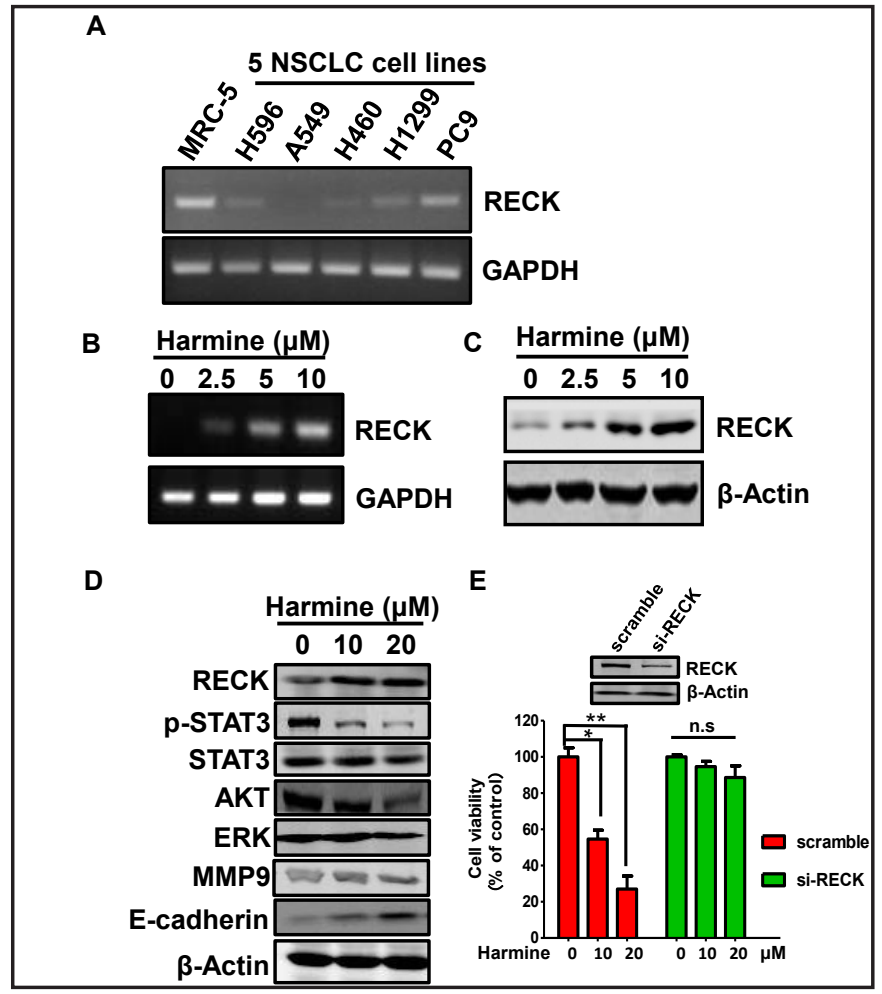

\section{KARGER}


knocking-down RECK gene by siRNAs (Fig. 5E). Furthermore, when explored the growth and metastasis related signal pathways by H\&E staining and immunohistochemistry analysis in A549luc orthotopic xenograft mice, we found the expression of RECK increased, while the expression of MMP9 decreased in Harmine-treated mice tumors, compared to the control group (Fig. 6). This finding suggests that Harmine treatment activated RECK activity and further suppressed the p-STAT3 and MMP9 pathway that led to the reversal of EMT in vivo.

\section{Discussion}

Lung cancer especially NSCLC causes more deaths than any other types of cancer in both men and women worldwide. NSCLC is typically diagnosed at advanced stages, the median survival time is approximately 8

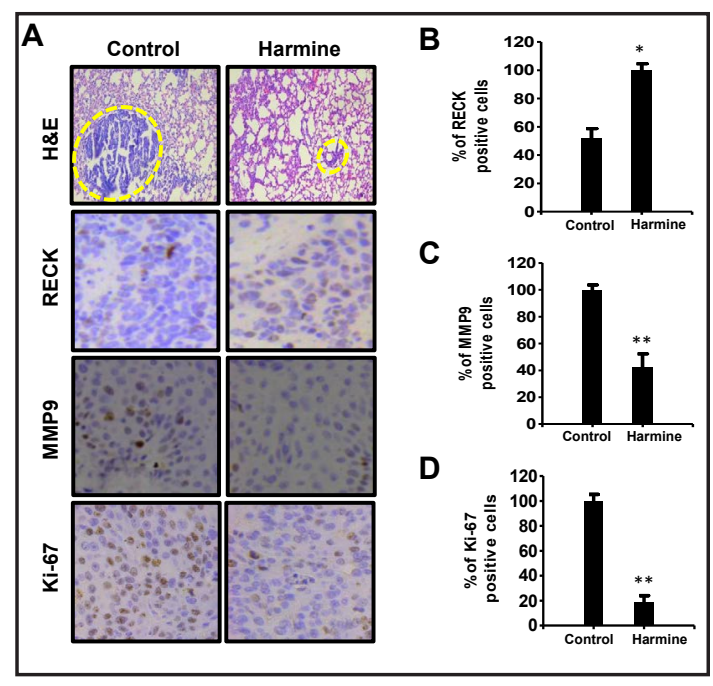

Fig. 6. (A) H\&E staining and immunohistochemical analysis of Ki-67, RECK and MMP9 in primary tumors from A549-luc orthotopic xenograft mice. (B) Quantitative analysis of (A). The means and 95\% confidence intervals (error bars) were presented $\left(* * * \mathrm{P}<0.001,{ }^{* *} \mathrm{P}<0.01,{ }^{*} \mathrm{P}<0.05\right)$. months. In the past few years, great strides have been made in the discovery of effective and new therapies for NSCLC. However, most NSCLC cases ultimately relapsed and progressed to intractable late-stage metastatic disease. Drug resistance and concomitant side effects hinder the clinical use for effective cancer therapy. Therefore, it is necessary to develop new agents that overcome these hurdles.

Natural sources provide beneficial pharmacotherapy for people worldwide. Over $70 \%$ of anti-tumor drugs developed in the last three decades are natural products. In this study, we reported for the effect of Harmine, a tricyclic compound from Peganum harmala, against NSCLC cells in a time- and dose-dependent manner. Harmine significantly inhibited NSCLC cell proliferation and growth in vitro as shown in Fig. 2. We also performed the cell viability assay for Harmine treatment on normal human lung cells, MRC-5 cells. The result demonstrated the selectivity of Harmine toward NSCLC cells (Fig. 2).

To further determine whether the anti-proliferative effect of Harmine is due to the inhibition of cell cycle progression or the induction of apoptosis, we carried out a flow cytometric analysis of the cell cycle in A549 cells. Our data showed Harmine altered the cell cycle distribution and arrested cells at G1/S phase (Fig. 2C). Interestingly, flow cytometric analysis from other study [31] demonstrated human colorectal carcinoma SW620 cells were arrested at S and G2 /M phase with Harmine treatment. This indicates that different types of tumor cell lines respond differently to Harmine's treatment, and multiple mechanisms might be responsible for cell cycle arrest in various cancer cells. Cell cycle arrest might be an upstream event leading to cell apoptosis. Under Harmine treatment, we indeed found activation of cellular apoptosis by using TUNEL staining for A549 cells (Fig. 2D). Importantly, in the in vitro cell migration and invasion assays, we found Harmine treatment led to significant efficacy on NSCLC cells (Fig. 3). In further in vivo study, we tested efficacy of Harmine on pre-established xenografts of A549 cells in nude mice. The administration of Harmine halted the growth and metastasis of human NSCLC xenograft derived from A549 cells (Fig. 4 and Fig. 6). Our work further proved that Harmine might exert its antitumor efficacy through the activation of RECK signaling pathway (Fig. 5). Harmine could directly up-regulate RECK's mRNA and protein (Fig. 5B and 5C), then inducing an impaired phosphorylation of STAT3, while not affected the total STAT3 protein level (Fig. 5D). When 


\section{Cellular Physiology Cell Physiol Biochem 2018:45:1807-1817

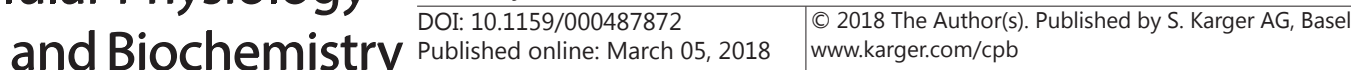 \\ Shen et al.: Harmine Suppresses NSCLC by Activating RECK Signaling}

STAT3 activation was inhibited by Harmine, the expression level of MMP9 was accordingly reduced (Fig. 5D). All these results contributed to the restrained metastasis of NSCLC.

In addition to Harmine's remarkable anticancer efficacy shown both in vitro and in vivo, another advantage of Harmine is its acceptable toxicity. Current commercial microtubule targeting agents are widely used in the clinic but side effects are significant due to drug toxicity. Although Harmine also showed some toxicity on MRC-5 cells, an immortalized human normal lung cell line, the effect was negligible compared to the effect on the cancer cells (Fig. 2A). Besides, in xenograft tumor model, in vivo treatment of Harmine at the effective concentration had little effect on the body weight of the mice (Fig. 4E).

\section{Conclusion}

In summary, we demonstrate Harmine is efficacious in suppressing the growth and metastasis of NSCLC cells both in vitro and in vivo via activating the RECK signaling pathway. These findings suggest Harmine is a novel candidate for a chemotherapeutic agent against NSCLC.

\section{Acknowledgements}

This work was supported by the National Natural Science Foundation of China (No.81602649), Hubei Provincial Natural Science Foundation of China (No. 2016CFB210) and the Startup Scientific Research Foundation for Doctors of Hubei University of Science and Technology (No. BK1506).

\section{Disclosure Statement}

The authors declare no competing financial interests.

\section{References}

$>1$ Li Y, Xu D, Bao CY, Zhang YN, Chen D, Zhao FY, Ding J, Liang LH, Wang QF, Liu L, Li JJ, Yao M, Huang SL, He XH: MicroRNA-135b, a HSF1 target, promotes tumor invasion and metastasis by regulating RECK and EVI5 in hepatocellular carcinoma. Oncotarget 2015;6:2421-2433.

-2 Hong KJ, Wu DC, Cheng KH, Chen LT, Hung WC: RECK Inhibits Stemness Gene Expression and Tumorigenicity of Gastric Cancer Cells by Suppressing ADAM-Mediated Notch1 Activation. J Cell Physiol 2014;229:191-201.

-3 Stenzinger A, von Winterfeld M, Rabien A, Warth A, Kamphues C, Dietel M, Weichert W, Klauschen F, Wittschieber D: Reversion-inducing cysteine-rich protein with Kazal motif (RECK) expression: an independent prognostic marker of survival in colorectal cancer. Hum Pathol 2012;43:1314-1321.

-4 Alexius-Lindgren M, Andersson E, Lindstedt I, Engstrom W: The RECK Gene and Biological Malignancy-Its Significance in Angiogenesis and Inhibition of Matrix Metalloproteinases. Anticancer Res 2014;34:38673873.

5 Hieronimus B, Pfohl J, Busch C, Graeve L: Expression and Characterization of Membrane-Type 4 Matrix Metalloproteinase (MT4-MMP) and its Different Forms in Melanoma. Cell Physiol Biochem 2017;42:198210.

-6 Dong QZ, Zhao Y, Tan ST, Wang EH: Derlin-1 overexpression confers poor prognosis in muscle invasive bladder cancer and contributes to chemoresistance and invasion through PI3K/AKT and ERK/MMP signaling. Oncotarget 2017;8:17059-17069. 


\section{Cellular Physiology Cell Physiol Biochem 2018;45:1807-1817 \begin{tabular}{l|l} 
and Biochemistry Published online: March 05, 2018 & $\begin{array}{l}\text { (c) } 2018 \text { The Author(s). Published by S. Karger AG, Basel } \\
\text { www.karger.com/cpb }\end{array}$
\end{tabular}}

Shen et al.: Harmine Suppresses NSCLC by Activating RECK Signaling

7 Song N, Liu H, Ma XX, Zhang SL: Placental Growth Factor Promotes Metastases of Ovarian Cancer Through MiR-543-Regulated MMP7. Cell Physiol Biochem 2015;37:1104-1112.

8 Discacciati MG, Gimenes F, Pennacchi PC, Faiao-Flores F, Zeferino LC, Derchain SM, Teixeira JC, Costa MC, Zonta M, Termini L, Boccardo E, Longatto-Filho A, Consolaro ME, Villa LL, Maria-Engler SS: MMP-9/RECK Imbalance: A Mechanism Associated with High-Grade Cervical Lesions and Genital Infection by Human Papillomavirus and Chlamydia trachomatis. Cancer epidemiology, biomarkers \& prevention : a publication of the American Association for Cancer Research, cosponsored by the American Society of Preventive Oncology 2015;24:1539-1547.

-9 Chai EZP, Shanmugam MK, Arfuso F, Dharmarajan A, Wang C, Kumar AP, Samy RP, Lim LHK, Wang LZ, Goh BC, Ahn KS, Hui KM, Sethi G: Targeting transcription factor STAT3 for cancer prevention and therapy. Pharmacol Therapeut 2016;162:86-97.

10 Bosch-Barrera J, Menendez JA: Silibinin and STAT3: A natural way of targeting transcription factors for cancer therapy. Cancer Treat Rev 2015;41:540-546.

11 Siveen KS, Sikka S, Surana R, Dai XY, Zhang JW, Kumar AP, Tan BKH, Sethi G, Bishayee A: Targeting the STAT3 signaling pathway in cancer: Role of synthetic and natural inhibitors. Bba-Rev Cancer 2014;1845:136-154.

12 Wu K, Shen B, Jiang F, Xia L, Fan T, Qin M, Yang L, Guo J, Li Y, Zhu M, Du J, Liu Y: TRPP2 Enhances Metastasis by Regulating Epithelial-Mesenchymal Transition in Laryngeal Squamous Cell Carcinoma. Cell Physiol Biochem 2016;39:2203-2215.

13 Walsh LA, Roy DM, Reyngold M, Giri D, Snyder A, Turcan S, Badwe CR, Lyman J, Bromberg J, King TA, Chan TA: RECK controls breast cancer metastasis by modulating a convergent, STAT3-dependent neoangiogenic switch. Oncogene 2015;34:2189-2203.

14 Li JW, Jiang KL, Zhao FJ: Icariin regulates the proliferation and apoptosis of human ovarian cancer cells through microRNA-21 by targeting PTEN, RECK and Bcl-2.Oncol Rep 2015;33:2829-2836.

$\checkmark 15$ Ahn MY, Kang DO, Na YJ, Yoon S, Choi WS, Kang KW, Chung HY, Jung JH, Min DS, Kim HS: Histone deacetylase inhibitor, apicidin, inhibits human ovarian cancer cell migration via class II histone deacetylase 4 silencing. Cancer Lett 2012;325:189-199.

16 Torre LA, Bray F, Siegel RL, Ferlay J, Lortet-Tieulent J, Jemal A: Global Cancer Statistics, 2012 Ca-a Cancer Journal for Clinicians 2015;65:87-108.

17 Song GS, Shen J, Jiang FR, Hu RG, Li WY, An L, Zou RJ, Chen ZG, Qin ZY, Hu JQ: Hydrophilic Molybdenum Oxide Nanomaterials with Controlled Morphology and Strong Plasmonic Absorption for Photothermal Ablation of Cancer Cells. Acs Appl Mater Inter 2014;6:3915-3922.

18 Shen J, Ma H, Zhang T, Liu H, Yu L, Li G, Li H, Hu M: Magnolol Inhibits the Growth of Non-Small Cell Lung Cancer via Inhibiting Microtubule Polymerization. Cell Physiol Biochem 2017;42:1789-1801.

19 Shen J, Song GS, An M, Li XQ Wu N, Ruan KC, Hu JQ, Hu RG: The use of hollow mesoporous silica nanospheres to encapsulate bortezomib and improve efficacy for non-small cell lung cancer therapy. Biomaterials 2014;35:316-326.

20 Li C, Hu JQ, Li WY, Song GS, Shen J: Combined bortezomib-based chemotherapy and p53 gene therapy using hollow mesoporous silica nanospheres for p53 mutant non-small cell lung cancer treatment. Biomater SciUk 2017;5:77-88.

-21 Shen J, Sheng X, Chang Z, Wu Q Xie D, Wang F, Hu R: The heme-p53 interaction: Linking iron metabolism to p53 signaling and tumorigenesis. Mol Cell Oncol 2016;3:e965642.

-22 Zhang XF, Sun RQ, Jia YF, Chen Q, Tu RF, Li KK, Zhang XD, Du RL, Cao RH: Synthesis and mechanisms of action of novel harmine derivatives as potential antitumor agents. Sci Rep-Uk 2016;6

23 Gao J, Zhu H, Wan H, Zou X, Ma X, Gao G: Harmine suppresses the proliferation and migration of human ovarian cancer cells through inhibiting ERK/CREB pathway. Oncol Rep 2017

24 Pesta M, Kulda V, Topolcan O, Safranek J, Vrzalova J, Cerny R, Holubec L: Significance of methylation status and the expression of RECK mRNA in lung tissue of patients with NSCLC. Anticancer research 2009;29:4535-4539.

25 Takenaka K, Ishikawa S, Kawano Y, Yanagihara K, Miyahara R, Otake Y, Morioka Y, Takahashi C, Noda M, Wada H, Tanaka F: Expression of a novel matrix metalloproteinase regulator, RECK, and its clinical significance in resected non-small cell lung cancer. Eur J Cancer 2004;40:1617-1623. 


\section{Cellular Physiology Cell Physiol Biochem 2018;45:1807-1817

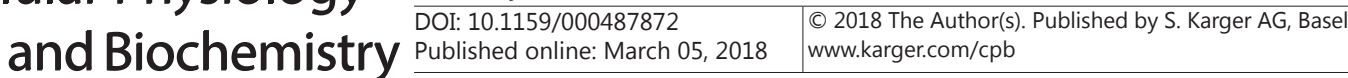

-26 Takenaka K, Ishikawa S, Yanagihara K, Miyahara R, Hasegawa S, Otake Y, Morioka Y, Takahashi C, Noda M, Ito H, Wada H, Tanaka F: Prognostic significance of reversion-inducing cysteine-rich protein with Kazal motifs expression in resected pathologic stage IIIA N2 non-small-cell lung cancer. Annals of surgical oncology 2005;12:817-824.

27 Shen J, Sheng XP, Chang ZN, Wu Q, Wang S, Xuan ZL, Li D, Wu YL, Shang YJ, Kong XT, Yu L, Li L, Ruan KC, Hu HY, Huang Y, Hui LJ, Xie D, Wang FD, Hu RG: Iron Metabolism Regulates p53 Signaling through Direct Heme-p53 Interaction and Modulation of p53 Localization, Stability, and Function. Cell Rep 2014;7:180193.

-28 Shen J, Zhang SP, Li Y, Zhang W, Chen JD, Zhang MR, Wang T, Jiang L, Zou XQ Wong JM, Li XT, Cui YP, Wang CG: p14(ARF) inhibits the functions of adenovirus E1A oncoprotein. Biochem J 2011;434:275-285.

29 Hu MC, Peng SH, He YD, Qin M, Cong XN, Xing YJ, Liu MY, Yi ZF: Lycorine is a novel inhibitor of the growth and metastasis of hormone-refractory prostate cancer. Oncotarget 2015;6:15348-15361.

30 Li J, Deng H, Hu M, Fang Y, Vaughn A, Cai X, Xu L, Wan W, Li Z, Chen S, Yang X, Wu S, Xiao J: Inhibition of non-small cell lung cancer (NSCLC) growth by a novel small molecular inhibitor of EGFR. Oncotarget 2015;6:6749-6761.

31 Guo JM, Kang GZ, Xiao BX, Liu DH, Zhang S: Effect of daidzein on cell growth, cell cycle, and telomerase activity of human cervical cancer in vitro. Int J Gynecol Cancer 2004;14:882-888. 